\title{
Generalized Performance of Concatenated Quantum Codes-A Dynamical Systems Approach
}

\author{
Jesse Fern, Julia Kempe, Slobodan N. Simić, and Shankar Sastry, Fellow, IEEE
}

\begin{abstract}
We apply a dynamical systems approach to concatenation of quantum error correcting codes, extending and generalizing the results of Rahn et al. to both diagonal and nondiagonal channels. Our point of view is global: instead of focusing on particular types of noise channels, we study the geometry of the coding map as a discrete-time dynamical system on the entire space of noise channels. In the case of diagonal channels, we show that any code with distance at least three corrects (in the infinite concatenation limit) an open set of errors. For Calderbank-Shor-Steane (CSS) codes, we give a more precise characterization of that set. We show how to incorporate noise in the gates, thus completing the framework. We derive some general bounds for noise channels, which allows us to analyze several codes in detail.
\end{abstract}

Index Terms-Quantum channels, quantum error corrections, quantum fault tolerance.

\section{INTRODUCTION}

$\mathbf{I}$ $\mathrm{N}$ THIS PAPER, we analyze quantum codes in essence, abstracting their details as codes and extracting their fault tolerance properties using a dynamical systems approach. This framework has been initiated by Rahn et al. [1]. They show how to incorporate diagonal noise on the qubit into an effective channel on the logical qubits.

We broaden this viewpoint and extend their approach in several ways. We look at the effective channel from a dynamical systems point of view, using tools and methods from this field. In particular we characterize the region of correctable errors using tools from the analysis of fixed points and show how to incorporate perturbations of the coding map.

\footnotetext{
Manuscript received October 21, 2004; revised September 6, 2005. Recommended by Associate Editor A. Garulli. The work of J. Fern and J. Kempe was supported by the Defense Advanced Research Projects Agency (DARPA) and Air Force Laboratory, Air Force Material Command, USAF, under agreement F30602-01-2-0524, by DARPA and the Office of Naval Research under Grant FDN-00014-01-1-0826. The work of J. Fern, J. Kempe, and S. N. Simić was supported in part by the National Science Foundation ITR under Grant CCF-0205641. The work of J. Kempe was supported by ACI Sécurité Informatique, 2003-n24, project "Réseaux Quantiques," ACI-CR 2002-40 and the EU Fifth Framework Program RESQ IST-2001-37559.

J. Fern is with the Department of Mathematics, the University of California, Berkeley, CA 94720-3840 USA (e-mail: jesse@ math.berkeley.edu).

J. Kempe is with the Computer Science Division and Department of Chemistry, the University of California, Berkeley, CA 94720-3840 USA, and also with CNRS-LRI UMR 8623, Université de Paris-Sud, 91405 Orsay, France.

S. N. Simić was with the Department of Electrical Engineering and Computer Science, the University of California, Berkeley, CA 94720-3840 USA. He is now with the Department of Mathematics, San Jose State University, San Jose, CA 95192-0103 USA (e-mail: simic@ math.sjsu.edu).

S. Sastry is with the Department of Electrical Engineering and Computer Science, the University of California, Berkeley, CA 94720-3840 USA.

Digital Object Identifier 10.1109/TAC.2006.871942
}

Our second chain of results extends the results of [1] to the realistic model of faulty gates and general channels. Rahn et al. only analyzed the depolarizing channel on the physical qubits as the single source of noise. We show that incorporating noisy gates gives rise to a perturbed effective channel. We also analyze general noise on the qubits and give several bounds for the convergence of nondiagonal channels to diagonal channels. Our results are supported by several examples for the family of CSS-codes, which is the encoding predominantly proposed for fault-tolerant quantum computing. We simplify our bounds in the case of CSS codes and analyze the [[7,1,3]] code, the smallest member of the CSS family, in great detail.

1) Structure of the Paper: We first introduce the dynamical systems approach in Section II and establish the notation and some basics. In Section III, we extend this approach to diagonal channels, including an analysis of regions of convergence. Section IV deals with faulty gates. In Section V, we establish several results and examples for nondiagonal (i.e., general) noise channels and in Section VI, we discuss a way to improve channels. Our approach allows to drastically reduce the number of parameters, lending quantum error correcting codes (QECCs) to an elegant analysis. This, however, comes at some price, and in Section VII we outline some of the shortcomings of this approach, before concluding with some open questions.

\section{NOTATION AND FRAMEWORK}

In this section, we formulate the basic framework and review the main results from [1], which should be consulted for details. Quantum states are represented by their density matrices.

The error correction process consists of three parts: encoding $\mathcal{E}$, noise $\mathcal{N}$, and decoding $\mathcal{D}$. Each part is modeled as a quantum channel, namely, a map taking density matrices to density matrices. Quantum channels are required to be linear, trace-preserving, and completely positive, hence of the form

$$
\rho \rightarrow \sum_{j} A_{j} \rho A_{j}^{\dagger}, \quad \text { with } \quad \sum_{j} A_{j}^{\dagger} A_{j}=I
$$

where $A_{j}$ are linear operators and $I$ is the identity (cf. [2]). In addition, we will assume that the channels are time-independent in order to simplify the study of their convergence. In the subsequent sections, we will often denote quantum channels by $\$$.

Encoding $\mathcal{E}$ takes an initial logical qubit state $\rho_{0}$ to the initial register state $\rho(0)$ which evolves according to some continuous-time noise dynamics. We consider the evolution for a fixed amount of time $t$, turning noise into a discrete-time operation $\mathcal{N}$ 
which takes $\rho(0)$ into a final register state $\rho(t)=\mathcal{N}(\rho(0))$. Finally, decoding $\mathcal{D}$ takes $\rho(t)$ to the final logical qubit state $\rho_{f}$. The map

$$
\mathcal{G}=\mathcal{D} \circ \mathcal{N} \circ \mathcal{E}: \rho_{0} \rightarrow \rho_{f}
$$

describes the effective dynamics of the encoded information resulting from the physical dynamics of $\mathcal{N}$ and is called the effective channel.

We consider noise models $\mathcal{N}$ on $n$ qubits consisting of uncorrelated noise $\mathcal{N}^{(1)}$ on each single physical qubit, so

$$
\mathcal{N}=\overbrace{\mathcal{N}^{(1)} \otimes \ldots \otimes \mathcal{N}^{(1)}}^{n \text { times }} .
$$

Given an $n$ qubit quantum error correcting code $C$ with encoding operation $\mathcal{E}$ and decoding operation $\mathcal{D}$, the map taking the single qubit noise $\mathcal{N}^{(1)}$ to the effective channel $\mathcal{G}$

$$
\Omega^{C}: \mathcal{N}^{(1)} \rightarrow \mathcal{D} \circ\left(\mathcal{N}^{(1)}\right)^{\otimes n} \circ \mathcal{E}
$$

is called the coding map of $C$.

The density matrix of one qubit can be expanded in the standard Pauli basis $\mathcal{P}=\{I, X, Y, Z\}$ for density matrices and represented as a four-dimensional real vector. A noise channel $\mathcal{N}^{(1)}$ can then be represented as a $4 \times 4$ matrix

$$
\mathcal{N}^{(1)}=\left(\begin{array}{cccc}
1 & 0 & 0 & 0 \\
N_{X I} & N_{X X} & N_{X Y} & N_{X Z} \\
N_{Y I} & N_{Y X} & N_{Y Y} & N_{Y Z} \\
N_{Z I} & N_{Z X} & N_{Z Y} & N_{Z Z}
\end{array}\right)
$$

Zeroes in the first row are due to trace preservation. For an arbitrary $n$ qubit code $C$, the entries of the matrix $\mathcal{G}=\Omega^{C}\left(\mathcal{N}^{(1)}\right)$ can be calculated to be

$$
\mathcal{G}_{\sigma \sigma^{\prime}}=\sum_{\mu} \sum_{\nu} \beta_{\nu}^{\sigma} \alpha_{\mu}^{\sigma^{\prime}} \prod_{i=1}^{n} N_{\nu_{i} \mu_{i}}
$$

where $\mu=\left(\mu_{1}, \ldots, \mu_{n}\right), \nu=\left(\nu_{1}, \ldots, \nu_{n}\right)$ run over $\mathcal{P}^{\otimes n}$, and $\alpha_{\mu}^{\sigma^{\prime}}, \beta_{\nu}^{\sigma}$ are the coefficients in the expansions for the encoding and decoding operations relative to $\mathcal{P} \otimes n$. See [1] for details.

If the matrix (3) is diagonal, $\mathcal{N}^{(1)}$ is called a diagonal channel. In that case, we write $x=N_{X X}, y=N_{Y Y}$, and $z=N_{Z Z}$ and denote the channel by $[x, y, z]$. It was shown in [3] that complete positivity of such channels implies that the point $(x, y, z)$ must be in the tetrahedron $\Delta$ defined by

$$
\begin{array}{r}
-x+y+z \leq 1 \\
x-y+z \leq 1 \\
x+y-z \leq 1 \\
-x-y-z \leq 1 .
\end{array}
$$

It is easily checked that a single-bit Pauli channel with exclusive probabilities $0 \leq p_{X}, p_{Y}, p_{Z} \leq 1$

$\rho \rightarrow\left(1-p_{X}-p_{Y}-p_{Z}\right) \rho+p_{X} X \rho X+p_{Y} Y \rho Y+p_{Z} Z \rho Z$ has the following representation in the previous notation:

$$
\left[1-2\left(p_{Y}+p_{Z}\right), 1-2\left(p_{X}+p_{Z}\right), 1-2\left(p_{X}+p_{Y}\right)\right] .
$$

In fact, any diagonal channel can be realized as a single-bit Pauli channel, so the parametrizations of $\Delta$ via $[x, y, z]$ and via $\left(p_{X}, p_{Y}, p_{Z}\right)$ are equivalent.

The $n$ dimensional Pauli group is $\mathcal{P}_{n}=\{ \pm 1, \pm i\} \otimes \mathcal{P}^{\otimes n}$. Suppose we have a stabilizer code that encodes $k$ qubits into $n$. Its stabilizer $S$ is an abelian subgroup of $\mathcal{P}_{n}$ with $n-k$ generators $g_{i}$. The $2^{k}$-dimensional codespace is defined as

$$
C_{S}=\left\{|\psi\rangle \in\left(\mathbb{C}^{2}\right)^{\otimes n} \text { so that } g|\psi\rangle=|\psi\rangle \text { for all } g \in S\right\} \text {. }
$$

The subset of $\mathcal{P}_{n}$ that commutes with $S$ is the centralizer, and it includes encoded operations we can perform on the codespace. We measure each generator $g_{i}$, and let $\beta_{i}=0$ if we project into the +1 eigenspace, and $\beta_{i}=1$ if we project into the -1 eigenspace. We then have an error syndrome $\beta \in F_{2}^{n-k}$, and we correct with a recovery operator $R_{\beta} \in \mathcal{P}_{n}$.

It was shown in [1] that if $C$ is a stabilizer code, then $\Omega^{C}$ takes diagonal channels to diagonal channels. In fact, if $S_{1}, \ldots, S_{m}$ are the generators of $C$, then

$$
\Omega^{C}[x, y, z]=\left[\Omega_{X}^{C}(x, y, z), \Omega_{Y}^{C}(x, y, z), \Omega_{Z}^{C}(x, y, z)\right]
$$

where

$$
\begin{aligned}
\Omega_{\sigma}^{C}[x, y, z] & =\frac{1}{m} \sum_{k=1}^{m} f_{k \sigma} x^{w_{X}\left(S_{k} \bar{\sigma}\right)} y^{w_{Y}\left(S_{k} \bar{\sigma}\right)} z^{w_{Z}\left(S_{k} \bar{\sigma}\right)} \\
f_{k \sigma} & =\sum_{j} \eta\left(S_{k}, R_{j}\right) \eta\left(R_{j}, \bar{\sigma}\right)
\end{aligned}
$$

and $\eta\left(\sigma, \sigma^{\prime}\right)= \pm 1$, if $\sigma \sigma^{\prime}= \pm \sigma^{\prime}$, for $\sigma, \sigma^{\prime} \in\{I, X, Y, Z\}$. Here, $w_{\sigma}$ denotes the $\sigma$-weight, $\bar{\sigma}$ is the encoded $\sigma$, and the $R_{j}$ denote recovery operators corresponding to the error syndromes. For later purposes, we extend $\eta$ as the natural homomorphism to the negative of the Pauli matrices by $\eta\left(-\sigma, \sigma^{\prime}\right)=$ $\eta\left(\sigma,-\sigma^{\prime}\right)=-\eta\left(\sigma, \sigma^{\prime}\right)=\eta\left(-\sigma,-\sigma^{\prime}\right)$.

Therefore, the components of $\Omega^{C}[x, y, z]$ are polynomials of degree $n$ in $x, y$, and $z$. Observe, however, that in general $\Omega^{C}$ is a map from a higher dimensional space of nondiagonal channels to itself. Nondiagonal channels of particular interest to us are unital channels; a channel $\mathcal{U}$ is unital if $\mathcal{U}(I)=I$.

An important result from [1] is that concatenation of codes translates into composition of coding maps. In other words, if $C_{1}$ and $C_{2}$ are codes and $C_{1} \circ C_{2}$ denotes their concatenation, then

$$
\Omega^{C}=\Omega^{C_{1}} \circ \Omega^{C_{2}} .
$$

Given a noise model $\mathcal{N}^{(1)}$ and code $C$, we are interested in what this noise looks like under repeated concatenation of the code $C$ with itself. Then the question is, does

$$
\Omega^{C^{\circ k}}\left(\mathcal{N}^{(1)}\right) \rightarrow I, \quad \text { as } k \rightarrow \infty ?
$$

If this is the case, $C$ corrects the error given by $\mathcal{N}^{(1)}$. 
Rahn et al. [1] focus mostly on the symmetric depolarizing channel given in the above notation by $\left[e^{-\gamma t}, e^{-\gamma t}, e^{-\gamma t}\right]$ and derive threshold estimates for various codes. We take a global point of view, where instead of looking at noise channels point by point, we consider the behavior of the coding map as a discrete-time dynamical system and study the set of all noise channels attracted to the identity channel under iteration of the coding map. This approach enables us to use methods from the theory of dynamical systems.

\section{OPEn Set of Correctable Diagonal ERrors}

We will first focus on diagonal noise channels, i.e., those given by a diagonal matrix, as discussed in the previous section. The standing assumption of this section is therefore that all noise channels are diagonal. We saw that we can characterize the asymptotic properties of the coding scheme involving the concatenation of a fixed code $C$ with itself by studying the long-term behavior of the dynamical system

$$
\Omega^{C}: \Delta \rightarrow \Delta
$$

We now review some necessary basics from the theory of dynamical systems. Good introductory references are [4] and [5].

\section{A. Dynamical Systems Preliminaries}

A (discrete-time) dynamical system is a map $f: M \rightarrow M$, where $M$ is a space with a certain additional structure (topological, metric, differentiable, etc.). In our case, it suffices to assume that $M$ is some Euclidean space $\mathbb{R}^{k}$ or a subset of it, and that $f$ is a differentiable map. We denote by $\mathbf{D} f(p)$ the derivative of $f$ at a point $p$ and think of it as a linear operator on $\mathbb{R}^{k}$. We will denote by $\|\mathbf{D} f(p)\|$ the norm of $\mathbf{D} f(p)$ as such on operator; that is

$$
\|\mathbf{D} f(p)\|=\max \{\|\mathbf{D} f(p) v\|:\|v\| \leq 1\} .
$$

(The norm on $\mathbb{R}^{k}$ is arbitrary but fixed.) If $\mathbf{D} f(p)$ depends differentiably on $p$, we define the second derivative of $f$ in the usual way as $\mathbf{D}^{2} f=\mathbf{D}(\mathbf{D} f)$; recall that $\mathbf{D}^{2} f(p)$ can be thought of a bilinear map $\mathbb{R}^{k} \times \mathbb{R}^{k} \rightarrow \mathbb{R}^{k}$ and $\left\|\mathbf{D}^{2} f(p)\right\|$ then denotes its norm. Continuing recursively, we say that $f$ is of class $C^{r}$ (or simply $C^{r}$ ) if $\mathbf{D}^{r} f(p)$ exists and is a continuous function of $p$.

For $p \in M$, the set $\left\{f^{n}(p): n=0,1,2, \ldots\right\}$, where $f^{n}=f \circ \cdots \circ f$ ( $n$ times), is called the orbit or trajectory of $f$. A fundamental question in the theory of dynamical systems is: what is the long term behavior of trajectories? That is, where does $f^{n}(p)$ end up eventually, as $n \rightarrow \infty$ ? The set of accumulation points of the orbit of $p$ is called the $\omega$-limit set of $p$. An example of such a set is a fixed point of $f$, i.e., a point $p$ such that $f(p)=p$. A fixed point $p$ is locally attracting if there exists a neighborhood $V$ of $p$ in $M$ such that for every $x \in V$, $f^{n}(x) \rightarrow p$, as $n \rightarrow \infty$. A basic criterion for a fixed point to be locally attracting is the following.

Lemma 3.1: Suppose $U \subset \mathbb{R}^{k}$ is open, $f: U \rightarrow \mathbb{R}^{k}$ is a $C^{1}$ map, $p \in U$ is a fixed point of $f$, and $\lambda_{0}=\|\mathbf{D} f(p)\|<1$. Then $p$ is locally attracting.
Proof: Let $\lambda_{0}<\lambda<1$. Since $\mathbf{D} f(x)$ depends continuously on $x$ and $\|\mathbf{D} f(p)\|<1$, there exists a neighborhood $V$ of $p$ in $U$ such that $\|\mathbf{D} f(x)\| \leq \lambda$, for all $x \in V$. Then, by the mean value theorem

$$
\|f(x)-f(p)\| \leq \lambda\|x-p\|
$$

for all $x \in V$. Therefore,

$$
\begin{aligned}
\left\|f^{n}(x)-p\right\| & =\left\|f^{n}(x)-f^{n}(p)\right\| \\
& \leq \lambda^{n}\|x-p\| \\
& \rightarrow 0
\end{aligned}
$$

as $n \rightarrow \infty$.

The largest such set $V$ is called the basin of attraction of the fixed point $p$, denoted by $\mathcal{B}(p)$. Let $B(x, r)$ denote the open ball of radius $r$ centered at $x$.

Lemma 3.2: Assume $f$ is $C^{2}$, the hypotheses of the previous lemma are satisfied, and $\left\|\mathbf{D}^{2} f(x)\right\| \leq K$, for all $x \in U$. Then, $B\left(p,\left(1-\lambda_{0}\right) / K\right) \cap U \subset \mathcal{B}(p)$.

Proof: The proof goes along similar lines as the previous one. Let $\lambda_{0}<\lambda<1$ be arbitrary and $0<r<\left(\lambda-\lambda_{0}\right) / K$. For an arbitrary point $x$ in the closed ball $B[p, r] \cap U$, we have

$$
\begin{aligned}
\|\mathbf{D} f(x)\| & \leq\|\mathbf{D} f(x)-\mathbf{D} f(p)\|+\|\mathbf{D} f(p)\| \\
& \leq K r+\lambda_{0} \\
& \leq \lambda
\end{aligned}
$$

that is, $f$ is a contraction on $B[x, r] \cap U$. Furthermore, for all $x \in B[p, r] \cap U$

$$
\begin{aligned}
\|f(x)-p\| & =\|f(x)-f(p)\| \\
& \leq \lambda\|x-p\| \\
& \leq r
\end{aligned}
$$

which implies that $B[p, r] \cap U$ is $f$-invariant. Therefore, under iteration of $f$, every point in $B[p, r] \cap U$ converges to $p$, so $B[p, r] \cap U \subset \mathcal{B}(p)$. Taking the union over all $\lambda \in\left(\lambda_{0}, 1\right)$ proves the claim.

Now take $f=\Omega^{C}$ and observe that $[1,1,1]$ is always an isolated fixed point of $\Omega^{C}$, though not necessarily attracting. For instance, $[1,1,1]$ is a saddle for the coding map $\Omega^{\mathrm{bf}}$ of the bit-flip code. However, if $C$ is the Shor or five-bit code, then $\mathbf{D} \Omega^{C}[1,1,1]=\mathbf{0}$, so $[1,1,1]$ is locally attracting. The following result shows that this is not a coincidence.

Proposition 3.3: Under the assumptions above, if $C$ is a quantum error correcting code of distance $\geq 3$, then

$$
\mathbf{D} \Omega^{C}[1,1,1]=\mathbf{0} .
$$

Proof: It suffices to show that $\mathbf{D} \Omega^{C}$ sends three linearly independent vectors to zero.

Since the distance of the code is at least three, $C$ corrects all errors of weight one. In particular, it corrects all single-bit Pauli channel errors

$$
\rho \rightarrow(1-\varepsilon) \rho+\varepsilon \sigma \rho \sigma
$$


for $\sigma \in\{X, Y, Z\}$ and $0 \leq \epsilon \leq 1$. Such errors correspond to noise channels $[1,1-2 \epsilon, 1-2 \epsilon],[1-2 \epsilon, 1,1-2 \epsilon]$, and $[1-2 \epsilon, 1-2 \epsilon, 1]$, for $\sigma=X, Y, Z$, respectively. Let us consider $\sigma=X$. To say that $C$ corrects $X$-errors means that

$$
\Omega^{C}[1,1-2 \varepsilon, 1-2 \varepsilon]=\left[1,1-O\left(\varepsilon^{2}\right), 1-O\left(\varepsilon^{2}\right)\right] .
$$

This implies that the directional derivative

$$
\mathbf{D} \Omega^{C}[1,1,1] v_{X}=\left.\frac{d}{d \varepsilon}\right|_{\varepsilon=0} \Omega^{C}\left([1,1,1]+\varepsilon v_{X}\right)=0
$$

where $v_{X}=(0,-1,-1)^{T}$. Similarly, we can show that $\mathbf{D} \Omega^{C}[1,1,1] v_{Y}=\mathbf{D} \Omega^{C}[1,1,1] v_{Z}=0$, where $v_{Y}=(-1,0,-1)^{T}$ and $v_{Z}=(-1,-1,0)^{T}$. Since $v_{X}, v_{Y}, v_{Z}$ are linearly independent, it follows that $\mathbf{D} \Omega^{C}[1,1,1]=\mathbf{0}$.

Corollary 3.4: For every code $C$ of distance at least three, $[1,1,1]$ is an attracting fixed point of the coding map $\Omega^{C}: \Delta \rightarrow$ $\Delta$. If $\mathcal{B}_{C}$ denotes its basin of attraction and $\left\|\mathbf{D}^{2} \Omega^{C}\right\| \leq K$ on $\Delta$, then

$$
B\left([1,1,1], \frac{1}{K}\right) \cap \Delta \subset \mathcal{B}_{C} .
$$

Proof: Observe that $\Omega^{C}$ can be extended to the whole space $\mathbb{R}^{3}$, has $[1,1,1]$ as a fixed point, and, by Proposition 3.3 , $\lambda_{0}=\mathbf{D} \Omega^{C}[1,1,1]=\mathbf{0}$. Therefore, $[1,1,1]$ is locally attracting for $\Omega^{C}$ as a map $\mathbb{R}^{3} \rightarrow \mathbb{R}^{3}$. By Lemma $3.2, B([1,1,1], 1 / K)$ is contained in the basin of attraction of $[1,1,1]$, again as a fixed point of $\Omega^{C}: \mathbb{R}^{3} \rightarrow \mathbb{R}^{3}$. However, we know that $\Delta$ is an invariant set for $\Omega^{C}$, i.e., $\Omega^{C}(\Delta) \subset \Delta$, and it contains $[1,1,1]$. Therefore, points in $B([1,1,1], 1 / K) \cap \Delta$ are both attracted to $[1,1,1]$ and stay in $\Delta$ under iteration of $\Omega^{C}$. This proves (7).

Proposition 3.5: Suppose $C$ is a CSS code. It will be shown in Theorem 5.7 that

$$
\Omega^{C}[x, y, z]=[f(x), g(x, y, z), f(z)]
$$

for some polynomials $f, g$. Let $a$ be the largest fixed point of $f$ in $(0,1)$. Then

$$
\mathcal{B}_{C}=\{[x, y, z] \in \Delta: x>a, z>a\}
$$

Proof: It follows from Proposition 3.3. that 1 is an attracting fixed point of $f$. Let $(\alpha, \beta)$ be its basin of attraction. It is well known that its boundary $\{\alpha, \beta\}$ is $f$-invariant. Since $\alpha \in[a, 1)$ and $[a, 1)$ is $f$-invariant, it follows that $\alpha$ is a fixed point of $f$. Therefore, $\alpha=a$. This means that for every $x \in$ $(a, 1), f^{k}(x) \rightarrow 1$, as $k \rightarrow \infty$.

Now suppose $[x, y, z] \in \Delta, x>a, z>a$. Then

$$
\left(\Omega^{C}\right)^{k}[x, y, z]=\left[f^{k}(x), y_{k}, f^{k}(z)\right]
$$

We know that $f^{k}(x), f^{k}(z) \rightarrow 1$. Let $y_{*}$ be an accumulation point of the sequence $\left(y_{k}\right)$. Since $\left[1, y_{*}, 1\right] \in \Delta$, it follows that $y_{*}=1$. Therefore, $\left(\Omega^{C}\right)^{k}[x, y, z] \rightarrow[1,1,1]$, as $k \rightarrow \infty$, which implies $\{[x, y, z] \in \Delta: x>a, z>a\} \subseteq \mathcal{B}_{C}$.

To show the opposite inclusion, assume the contrary, i.e., that there exists a point $p=[x, y, z] \in \mathcal{B}_{C}$ such that $p \notin\{[x, y, z] \in$ $\Delta: x>a, z>a\}$. Then $x \leq a$ or $z \leq a$. In the former case, $f^{k}(x)$ does not converge to 1 , and in the latter, $f^{k}(z) \nrightarrow 1$, contrary to our assumption that $p$ is in the basin of attraction of $[1,1,1]$.

\section{FAulty Gates}

We want to extend the analysis in [1] to include faulty gate operations both in the error correction and in the computation circuits. Gate errors are a common form of noise in quantum information processing. We show how to incorporate faulty gates into the current framework and how they change the effective channel and the coding map. Note that fault tolerance for our noise model has been shown, but that there is some dispute about the validity of that model and whether quantum fault tolerance is possible [6].

\section{A. A Simple Noise Model}

Our first approach is to start with a very simple error model for faulty unitary gates $G$

$$
G: \rho \longrightarrow(1-\varepsilon) G \rho G^{\dagger}+\varepsilon \frac{1}{N} I .
$$

This error model is rather generic. It has the additional advantage that noise from sequential gates is additive; if we combine two faulty operations as in (8), we obtain

$$
\begin{aligned}
G_{2} \circ G_{1}: \rho \longrightarrow & G_{2}\left(\left(1-\varepsilon_{1}\right) G_{1} \rho G_{1}^{\dagger}+\frac{\varepsilon_{1}}{N} I\right) \\
= & \left(1-\varepsilon_{2}\right)\left(1-\varepsilon_{1}\right) G_{2} G_{1} \rho G_{1}^{\dagger} G_{2}^{\dagger} \\
& +\left(1-\varepsilon_{2}\right) \frac{\varepsilon_{1}}{N} I+\frac{\varepsilon_{2}}{N} I \\
\approx & \left(1-\varepsilon_{1}-\varepsilon_{2}\right) G_{2} G_{1} \rho\left(G_{2} G_{1}\right)^{\dagger} \\
& +\frac{\varepsilon_{1}+\varepsilon_{2}}{N} I
\end{aligned}
$$

i.e. a faulty process with $\epsilon=\epsilon_{1}+\epsilon_{2}$. As we have seen, the effective dynamics of one level of concatenation is simply encoding, noise and decoding, i.e.,

$$
\mathcal{G}=\mathcal{D} \circ \mathcal{N} \circ \mathcal{E}
$$

Let us also assume here that the noise on the qubits is unital, i.e. $\mathcal{N}(I)=I$. We now show that faulty gates in this model have the same effect as noise; hence, we can effectively treat noise from faulty gates and other types of noise on the qubits in the same way.

The encoding operation can be written concisely as $\mathcal{E}(\rho)=$ $B \rho B^{\dagger}$, where $B=|\overline{0}\rangle\langle 0|+| \overline{1}\rangle\langle 1|$ (or, for codes that encode more than one qubit, $\left.B=\sum_{i}|\bar{i}\rangle\langle i|\right)$. This encoding is performed by applying a sequence of gates, possibly faulty, as in (8). The operation corresponding to $B$ can be implemented with unitary gates in a larger space by appending some ancillary qubits, for instance as $U_{B}:|i\rangle|0\rangle \longrightarrow|\bar{i}\rangle$. If errors occur according to (8), the resulting operation will be $\mathcal{E}_{\epsilon_{E}}: \rho \rightarrow$ $\left(1-\epsilon_{E}\right) U_{B} \rho U_{B}^{\dagger}+\left(\epsilon_{E} / N\right) I=\left(1-\epsilon_{E}\right) \mathcal{E}(\rho)+\left(\epsilon_{E} / N\right) I$, where $\mathcal{E}$ denotes the error-free encoding and $\epsilon_{E}$ is the noise accumulated from gates during encoding. In an analogous way, it can be seen that a decoding map $\mathcal{D}$, implemented with faulty gates, can be written as $\mathcal{D}_{\epsilon_{D}}: \rho \rightarrow\left(1-\epsilon_{D}\right) \mathcal{D}(\rho)+\left(\epsilon_{D} / 2\right) I$, where we have used that $\mathcal{D}:(1 / N) I \longrightarrow(1 / 2) I$. Putting this together 
under the simplifying assumption that $\mathcal{N}(I)=I$ (unital channels), and using additivity of error from faulty gates, we get

$$
\rho \longrightarrow(1-\varepsilon) \mathcal{G}(\rho)+\frac{\varepsilon}{2} I
$$

where $\epsilon=\epsilon_{D}+\epsilon_{E}$ and $\mathcal{G}$ is the effective channel with perfect gates. In other words, faulty gates only contract the iterated map by $(1-\epsilon)$. As a result, the coding map $\Omega^{C}$ (see (2)) changes to $\Omega_{f}^{C}$, the coding map with faulty gates, as

$$
\Omega_{f}^{C}: \mathcal{N} \longrightarrow(1-\varepsilon) \mathcal{D} \circ \mathcal{N} \circ \mathcal{E}+\varepsilon \frac{1}{2} I=(1-\varepsilon) \Omega_{C}+\varepsilon \frac{1}{2} I .
$$

The entries of the matrix $\mathcal{G}$ for the coding map change as

$$
\mathcal{G}_{\sigma \sigma^{\prime}}^{f}=(1-\varepsilon) G_{\sigma \sigma^{\prime}}+\frac{\varepsilon}{2} \delta_{\sigma 1} \delta_{\sigma^{\prime} 1}
$$

where we have used the fact that the coding map whose only nonzero entry is $G_{11}$ represents a mapping of $\rho$ to the identity matrix. In other words, the incorporation of faulty gates into our analysis results in an affine mapping of the coding map: $G$ is contracted by $(1-\epsilon)$ and the element $\epsilon \delta_{11}$ is added.

\section{B. More General Noise}

It is not difficult to extend this analysis to more general noise in the gates and general noise on the qubits. Let us assume that instead of the restricted noise model of (8) we are dealing with generic noise of rate $\epsilon$. We can write

$$
G: \rho \longrightarrow(1-\varepsilon) G \rho G^{\dagger}+\varepsilon N_{G}(\rho)
$$

where $N_{G}$ is some general noise operation.

The analysis of the previous Section IV-A goes through line by line. The noise process is additive (with $I / N$ in (9) replaced by $\epsilon_{1} G_{2} N_{G_{1}}(\rho) G_{2}^{\dagger}+\epsilon_{2} N_{G_{2}}(\rho)$ ). The encoding and decoding operations can then be written as

$$
\begin{gathered}
\mathcal{E}_{\varepsilon_{E}}: \rho \rightarrow\left(1-\varepsilon_{E}\right) U_{B} \rho U_{B}^{\dagger}+\frac{\varepsilon_{E}}{N} I \\
=\left(1-\varepsilon_{E}\right) \mathcal{E}(\rho)+\varepsilon_{E} N_{E}(\rho) \\
\mathcal{D}_{\varepsilon_{D}}: \rho \rightarrow\left(1-\varepsilon_{D}\right) \mathcal{D}(\rho)+\varepsilon_{D} N_{D}(\rho)
\end{gathered}
$$

where $N_{E}$ and $N_{D}$ are the noise resulting from encoding, respectively, decoding. Concatenating yields

$$
\rho \longrightarrow(1-\varepsilon) \mathcal{G}(\rho)+\varepsilon N_{D E}
$$

with $\epsilon=\epsilon_{E}+\epsilon_{D}$ and the cumulative noise can be written to first order as

$$
\varepsilon N_{D E}=\varepsilon_{E} \mathcal{D}\left(\mathcal{N}\left(N_{E}(\rho)\right)\right)+\varepsilon_{D} N_{D}(\mathcal{N}(\mathcal{E}(\rho)) .
$$

The new coding map with faulty gates is then very similar to before

$\Omega_{f}^{C}: \mathcal{N} \longrightarrow(1-\varepsilon) \mathcal{D} \circ \mathcal{N} \circ \mathcal{E}+\varepsilon N_{D E}(\rho)=(1-\varepsilon) \Omega_{C}+\varepsilon N_{D E}$.

In other words, faulty gates introduce a perturbation to the original coding map studied in the previous section. They can be treated in the same way as noise on the qubits. In fact we see that the occurrence of faulty gates is the same as a process with increased noise on the gates and perfect gates. However, if the noise on gates is small compared to the noise on qubits, we can treat it as a perturbation to the original coding map. We will show how to incorporate such perturbations in the analysis with the following Lemma. Here, $\|h\| C^{1}$ denotes the $C^{1}$ norm of a smooth map $h$ on its domain, that is, the maximum of the suprema of $\|h\|$ and $\|\mathbf{D} h\|$.

Lemma 4.1: Suppose $U \subset \mathbb{R}^{n}$ is an open set, $f: U \rightarrow \mathbb{R}^{n}$ is smooth (at least $C^{2}$ ), $f(p)=p$ and $\lambda=\|\mathbf{D} f(p)\|<1$.Then for small enough $\epsilon>0$ and every smooth map $g: U \rightarrow \mathbb{R}^{n}$, if $\|g-f\|_{C^{1}}<\epsilon$, then $g$ has a fixed point $q$ such that $\|\mathbf{D} g(q)\|<1$ and $|q-p|<\epsilon /(1-\lambda)$.

In other words, if a map has an attracting fixed point, then any sufficiently small $C^{1}$ perturbation of it also has an attracting fixed point which is close to the original one.

This is a standard fact from the theory of dynamical systems; for completeness, we supply a proof here.

a) Proof: Let $M$ be an upper bound of $\left\|\mathbf{D}^{2} f\right\|$ on some relatively compact neighborhood $V$ of $p$. Since $\lambda<1$, there exists $r>0$ such that $f$ maps the closed ball $B[p, r]$ into itself and $B[p, r] \subset V$. Without loss, we can take $r$ so small that $r<(1-\lambda) / M$. Assume $0<\epsilon<\min ((1-\lambda) r, 1-\lambda-$ $M r)$. Then it is not difficult to show that for every $x \in B[p, r]$, $\|g(x)-p\| \leq \epsilon+\lambda r<r$, which means that $g$ takes $B[p, r]$ into itself. Therefore, by the Brouwer fixed point theorem, $g$ has a fixed point, say $q$, in $B[p, r]$. Since

$$
\begin{aligned}
|q-p| & =|g(q)-p| \\
& \leq|g(q)-f(q)|+|f(q)-p| \\
& \leq \varepsilon+\lambda|q-p|
\end{aligned}
$$

we obtain $|q-p|<\epsilon /(1-\lambda)$.

To show that $q$ is an attracting fixed point for $g$, let us show that $\|\mathbf{D} g(q)\|<1$. Observe first that $\|\mathbf{D} f(q)\| \leq M r+\lambda<1-$ $\epsilon$. Therefore, $\|\mathbf{D} g(q)\| \leq\|\mathbf{D} g(q)-\mathbf{D} f(q)\|+\|\mathbf{D} f(q)\|<1$.

It is clear from (10) that the coding map $\Omega_{f}^{C}$ of a code with faulty gates is a $C^{1}$ small perturbation of the coding map $\Omega^{C}$ with perfect gates.

\section{ANALYSIS OF CHANNELS}

In this section, we will give several technical results about channel maps, which we will subsequently use to analyze various diagonal and nondiagonal channels and to give examples. In particular, we will study in detail how nondiagonal elements of a noise channel affect its convergence and threshold.

\section{A. The Two-Point Theorem}

We look at bounds for a general channel, resulting in Theorem 5.4.

Lemma 5.1: For any nonidentity Pauli matrix $\sigma$

$$
\begin{aligned}
& N_{\sigma X}^{2}+N_{\sigma Y}^{2}+N_{\sigma Z}^{2} \leq\left(1-\left|N_{\sigma I}\right|\right)^{2} \\
& \left(N_{X I} \pm N_{X \sigma}\right)^{2}+\left(N_{Y I} \pm N_{Y \sigma}\right)^{2}+\left(N_{Z I} \pm N_{Z \sigma}\right)^{2} \leq 1
\end{aligned}
$$

All elements of the channel are real.

Proof: $\mathcal{N}$ preserves hermiticity, and is positive (sends nonnegative $\rho$ to nonnegative $\rho$ ) [7]. The first condition implies that the elements are real. Then the adjoint channel, which has the map $\mathcal{N}^{\dagger} \rho=\sum_{k} A_{k}^{\dagger} \rho A_{k}$, is also positive. A simple 
calculation shows that a matrix $\rho=c_{I} I+c_{X} X+c_{Y} Y+c_{Z} Z$ is nonnegative if and only if $c_{I} \geq \sqrt{c_{X}^{2}+c_{Y}^{2}+c_{Z}^{2}}$.

Let $c=\sqrt{N_{\sigma X}^{2}+N_{\sigma Y}^{2}+N_{\sigma Z}^{2}}$, and apply

$$
\mathcal{N}\left(c I \pm\left(N_{\sigma X} X+N_{\sigma Y} Y+N_{\sigma Z} Z\right)\right)
$$

which gives $c_{I}=c$, and $c_{\sigma}=c N_{\sigma I} \pm c^{2}$, so the nonnegative condition gives $\left|c N_{\sigma I} \pm c^{2}\right| \leq c$, from which we get $c^{2} \leq$ $\left(1-\left|N_{\sigma I)}\right|\right)^{2}$, which gives (11).

Let $b_{\sigma \sigma^{\prime}}=N_{X \sigma} N_{X \sigma^{\prime}}+N_{Y \sigma} N_{Y \sigma^{\prime}}+N_{Z \sigma} N_{Z \sigma /}$. Now let $c=\sqrt{b_{I I}+b_{\sigma \sigma} \pm 2 b_{I \sigma}}$. Then, apply $\mathcal{N}^{\dagger}$ to

$c I-\left(N_{X I} X+N_{Y I} Y+N_{Z I} Z\right) \pm\left(N_{X \sigma} X+N_{Y \sigma} Y+N_{Z \sigma} Z\right)$

which gives $c_{I}=c-b_{I I} \pm b_{I \sigma}$ and $c_{\sigma}=-b_{I \sigma} \pm b_{\sigma \sigma}$, so $c-b_{I I} \pm b_{I \sigma} \geq\left|-b_{I \sigma} \pm b_{\sigma \sigma}\right|$, which gives $c \geq b_{I I}+b_{\sigma \sigma} \pm$ $2 b_{I \sigma}=c^{2}$, so $c \leq 1$, which gives (12).

This proof extends naturally to multi-qubit channels.

Corollary 5.2: Each row of a quantum channel $\mathcal{N}$ in the Pauli basis has norm at most 1 .

Proof: Since $\left|N_{\sigma I}\right| \leq 1$, we have $1-N_{\sigma I}^{2} \geq\left(1-\left|N_{\sigma I}\right|\right)^{2}$, and so the result follows from (11).

Corollary 5.3: Let $A=N_{X I}^{2}+N_{Y I}^{2}+N_{Z I}^{2}$ be the nonunital portion of the channel. Then, we have that any other column of the channel in the Pauli basis has $L_{2}$ norm squared $N_{X \sigma}^{2}+$ $N_{Y \sigma}^{2}+N_{Z \sigma}^{2} \leq 1-A$.

Proof: Follows immediately from (12).

Theorem 5.4 (Two-Point Theorem): If two of $N_{X X}, N_{Y Y}$, $N_{Z Z}$ are 1, then the channel is the identity channel.

Proof: Let $\sigma_{1}, \sigma_{2}, \sigma_{3}$ be some permutation of the Pauli matrices such that $N_{\sigma_{1} \sigma_{1}}=N_{\sigma_{2} \sigma_{2}}=1$. From Corollary 5.2, $N_{\sigma_{1} \sigma_{1}}$ and $N_{\sigma_{2} \sigma_{2}}$ are the only nonzero elements in their rows. From Corollary 5.3, the nonunital part must be 0 , and $N_{\sigma_{1} \sigma_{1}}$ and $N_{\sigma_{2} \sigma_{2}}$ are the only nonzero elements in their columns. It then follows that the channel is diagonal. From the conditions on diagonal channels given in (5), it easily follows that if two terms are equal to 1 , the third term must equal 1 , and so we have the identity channel.

\section{B. Generalized Shor Codes}

In this section, we give a first application of our formalism and the general bounds we obtained. We study generalized Shor codes, which are bit flip and phase flip codes concatenated with each other. We will assume a diagonal channel $[x, y, z]$ in what follows. Note that Theorem 5.4 is easy to prove in this case; it follows immediately from (5).

1) Bit Flip, Phase Flip: The $n$ qubit bit flip code is a classical code on $n$ qubits that corrects all bit flip errors on less than $n / 2$ qubits and none of the errors on greater than $n / 2$ qubits; if $n$ is even it also corrects half of the errors on exactly $n / 2$ qubits. The coding map is $\Omega^{b f_{n}}[x, y, z]=\left[x^{n}, h_{n}(x, y, z), f_{n}(z)\right]$. To see this note that the code does not correct phase flips ( $Y$ or $Z$ errors), and so if $p=p_{Y}+p_{Z}$, the $p$-component of the coding map must be a function of only $p$. Since $x=1-2\left(p_{Y}+p_{Z}\right)=1-2 p$, it follows that the $x$-component of the coding map must be a function of only $x$. The only such element of the $X$ equivalence class gives us $x^{n}$.

To see that the $z$-component depends on $z$ only, note that the code can correct bit flips ( $X$ or $Y$ errors), sending them to $I$ or $Z$ errors, respectively, and so if $p^{\prime}=p_{X}+p_{Y}$, by similar reasoning as before we observe that the $p^{\prime}$ component depends only on $p^{\prime}$ and hence that the $z$-component is a function of only $z$. Now, assume only $X$ errors. Then $z=1-2 p_{X}$, and $f_{n}(z)=1-2 g(1-z / 2)$, where $g(p)$ is the failure probability as a function of an $X$ error rate of $p$. We can obtain $g(p)$ from the properties of the classical bit flip code.

Since the function $h_{n}(x, y, z)$ does not affect the $x$ and $z$ components of the channel, from Theorem 5.4, we may ignore it for the purposes of convergence to the identity channel.

Some values of $f_{n}$ are

$$
\begin{aligned}
& f_{1}(x)=f_{2}(x)=x \\
& f_{3}(x)=f_{4}(x)=\frac{3}{2} x-\frac{1}{2} x^{3} \\
& f_{5}(x)=f_{6}(x)=\frac{15}{8} x-\frac{5}{4} x^{3}+\frac{3}{8} x^{5} .
\end{aligned}
$$

For the phase flip code we get similarly $\Omega^{p f_{n}}[x, y, z]=$ $\left[f_{n}(x), h_{n}^{\prime}(x, y, z), z^{n}\right]$ by exchanging the roles of $x$ and $z$.

These codes will have two critical values, $x_{c}$ and $z_{c}$. If $x>x_{c}$ then $x \rightarrow 1$, and similarly for $z$.

2) Specific Codes: We can now obtain sharper results for the error threshold of concatenated bit flip and phase flip codes, extending [1].

The often discussed $[[9,1,3]]$ Shor code has the coding $\operatorname{map} \Omega^{\text {Shor }}[x, y, z]=\Omega^{p f_{3}} \Omega^{b f_{3}}[x, y, z]=$ $\left[f_{3}^{3}(x), h^{\prime \prime}(x, y, z), f_{3}\left(z^{3}\right)\right]$. We define a $[[25,1,5]]$ code to be $\Omega^{25}=\Omega^{p f_{5}} \Omega^{b f_{5}}$, and a $[[15,1,3]]$ code to be $\Omega^{15}=\Omega^{p f_{5}} \Omega^{b f_{3}}$

The [[25,1,5]] code has critical values of $x_{c}=0.916208$, and $z_{c}=0.645611$. The [[15,1,3]] code has critical values of $x_{c}=0.794438$ and $z_{c}=0.850432$. If $x=z$, the [[15,1,3]] code performs much better than the [[25,1,5]], even though it is less redundant.

\section{Convergence of Nondiagonal Channels}

In this section, we will establish some general results for nondiagonal channels in the case of stabilizer codes [8]. Nondiagonal channels are in general much harder to analyze than their diagonal counterparts, as the parameters span a 12-dimensional manifold. However, we will show that in certain cases these channels converge to diagonal channels, and will discuss when these converge to the identity channel.

We can decompose the single qubit noise operator $\mathcal{N}$ as

$$
\mathcal{N}=L+\varepsilon M
$$

where $L$ is the diagonal part, and $\epsilon$ is chosen such that $M$ has no term with absolute value more than 1 ; it contains the off-diagonal terms. We show that if $\epsilon$ is sufficiently small and $d \geq 3$, then repeated application of the coding map yields a diagonal matrix. This will allow to restrict our analysis to diagonal channels, at least in certain regimes.

We wish to analyze the absolute values of the difference that the nondiagonal terms make on the channel after we apply the coding map. Define the difference matrix

$$
\Gamma=\Omega^{C}(\mathcal{N})-\Omega^{C}(L) .
$$


Let us assume that the code is an $[[n, k, d]]$ stabilizer code [8] (it encodes $k$ qubits into $n$ qubits, and has distance $d$, which is the minimal weight of an undetected error). Let $m$ be the minimal weight of a nonidentity stabilizer element.

Theorem 5.5: The nondiagonal terms of the difference matrix $\Gamma$ have absolute value at most $c_{d} \epsilon^{d}$. The diagonal terms of $\Gamma$ are at most $c_{m} \epsilon^{m}$ in absolute value. These coefficients are bounded above by

$$
\max \left(c_{d}, c_{m}\right) \leq 2^{n-k} \sum_{\sigma^{\prime \prime}}\left|\mathcal{D}_{\sigma^{\prime} \sigma^{\prime \prime}}\right| \leq 4^{n-k}
$$

Proof: We can rewrite (4) as

$$
G_{\sigma \sigma^{\prime}}=\mathcal{D}_{\sigma} \mathcal{N} \mathcal{E}_{\sigma^{\prime}}
$$

where $\mathcal{E}_{\sigma}$ is the $\sigma$ column of $\mathcal{E}$ and similarly for $\mathcal{D}$. The (nonzero) entries of $\mathcal{E}_{I}$ are the stabilizer elements, and the nonzero elements of $\mathcal{E}_{\sigma}$ are $\bar{\sigma}$ times the stabilizer elements, where $\bar{\sigma}$ is the encoded $\sigma$. We note that $\mathcal{E}_{\sigma^{\prime} \sigma}$ is nonzero only if $\sigma /$ and $\bar{\sigma}$ are in the same equivalence class of $C(S)$ modulo $S$, where $S$ is the stabilizer group, and $C(S)$ is its centralizer (see [8] for more detailed definitions).

Now, the nondiagonal elements of $\Gamma$ depend on the nonzero elements of $\mathcal{E}_{\sigma}$ and $\mathcal{E}_{\sigma^{\prime}}$ with $\sigma \neq \sigma^{\prime}$, which correspond to the $\sigma$ and $\sigma^{\prime}$ equivalence classes of $C(S)$, which differ on at least $d$ qubits. Then from (4), respectively, (15), it follows that the nondiagonal terms involve at least $d$ nondiagonal terms of $\mathcal{N}$ and are hence $O\left(\epsilon^{d}\right)$ from (13). The difference of the diagonal elements corresponds to elements of the same $\mathcal{E}_{\sigma}$, which differ on at least $m$ qubits, since $m$ is the minimal weight of different elements in the same equivalence class (nonzero elements of the same $\left.\mathcal{E}_{\sigma}\right)$. Hence, they are $O\left(\epsilon^{m}\right)$.

From (15) it is easy to see that the coefficients $c_{d}$ and $c_{m}$ are bounded above by

$$
\sum_{\sigma^{\prime \prime}, \sigma^{\prime \prime \prime}}\left|\mathcal{D}_{\sigma \sigma^{\prime \prime}} \mathcal{E}_{\sigma^{\prime \prime \prime} \sigma^{\prime}}\right| \leq \sum_{\sigma^{\prime \prime}}\left|\mathcal{D}_{\sigma \sigma^{\prime \prime}}\right| \sum_{\sigma^{\prime \prime \prime}}\left|\mathcal{E}_{\sigma^{\prime \prime \prime} \sigma^{\prime}}\right| \leq 4^{n-k}
$$

where we used that each coefficient is at most 1 in absolute value and the cardinality of the stabilizer group.

Note that in certain cases we have explicit expressions for $\sum_{\sigma^{\prime \prime}}\left|\mathcal{D}_{\sigma^{\prime} \sigma^{\prime \prime}}\right|$, which can come from calculations with a diagonal noise channel and can give us tighter bounds on $c_{d}$ and $c_{m}$ than the generic $4^{n-k}$.

1) Convergence to the Identity: Suppose we concatenate the above coding map $i$ times. Then the absolute values of the offdiagonal terms are bounded above by $a_{i}$, where $a_{0}=\epsilon$, and $a_{n+1}=c_{d} a_{n}^{d}$. Then, from Theorem 5.5 where $\epsilon_{0}=\sqrt[d-1]{1 / c_{d}}$ is defined for $d>1$. Since these affect the diagonal terms by at most $c_{m} \epsilon^{m}$, we can bound the correction for the diagonal terms as

$$
b_{i}=c_{m} a_{i-1}^{m}=c_{m} c_{d}^{t \sum_{j=0}^{i-2} d^{j}} \varepsilon^{m d^{i-1}}=c_{m} \varepsilon_{0}^{m}\left(\frac{\varepsilon}{\varepsilon_{0}}\right)^{m d^{i-1}}
$$

Now we assume that the nondiagonal terms go to 0 , which means that $\epsilon<\epsilon_{0}$, and so $a_{i}$ and $b_{i}$ both go monotonically to 0 . From Theorem 5.5 , we can see that if the map $\Omega^{C}\left(L^{\otimes n}\right)-c_{m} \epsilon^{m} I$ converges to within $O(\epsilon m)$ of the identity matrix, then so does $\Omega^{C}\left(\mathcal{N}^{\otimes n}\right)$. However, we can get a tighter bound than this.

Let $L_{0}=\left[x_{0}, y_{0}, z_{0}\right]$ be the diagonal part of the channel. We define $L_{i}=\Omega^{C}\left(L_{i-1}\right)-b_{i} I$. We can think of the $L_{i}$ as a lower bound on the diagonal part of the channel. Then, the channel goes to $[1,1,1]$, if $L_{i} \rightarrow[1,1,1]$. These coding maps are $\Omega_{i}^{C}(L)=\Omega^{C}\left(L_{i-1}\right)-b_{i} I$, and $\Omega_{1}^{C}(L)=\Omega^{C}\left(L_{0}\right)-c_{m} \epsilon^{m} I$. The channel converges to identity if

$$
\ldots \circ \Omega_{2}^{C} \circ \Omega_{1}^{C} L=[1,1,1]
$$

\section{CSS Codes on 1 Qubit With a Generalized Noise Channel}

In this section, we tighten our result in the case of CSS codes [9]-[11].

Let our code be a $[[n, 1, d]]$ CSS code. From the construction of CSS codes from classical codes, $n$ must be odd. Its stabilizer group is generated by $n-1$ generators, half of which depend only on tensor products of $I \mathrm{~s}$ and $X \mathrm{~s}$, and the other half are the same, except they have $Z$ s replacing the $X \mathrm{~s}$. We can write the stabilizer group $S$ as the span of $\{S(X), S(Z)\}$, where $S(A) \in A_{S}$, and $A_{S}$ is the $n$-dimensional Pauli Matrices $\mathcal{P}_{n}$ which only depend on tensor products of $I$ and $A$. The stabilizer elements in $S(X)$ are used to correct against $Z$ errors, and the stabilizer elements of $S(Z)$ are used to correct against $X$ errors, and so we can write the set of recovery operators as $R\left(\varepsilon_{X}, Z\right)$ and $R\left(\varepsilon_{Z}, X\right)$, where $\varepsilon_{A}$ are the components of the syndromes obtained by measuring stabilizer generators from $S(A)$, and each $R(\varepsilon, A) \in A_{S}$.

The Pauli operators are encoded as

$$
\begin{aligned}
& \bar{X}=X^{\otimes n} \in X_{S} \\
& \bar{Z}=Z^{\otimes n} \in Z_{S} \\
& \bar{Y}=i \overline{X Z}=(-1)^{\frac{n-1}{2}} Y^{\otimes n} \in Y_{S} .
\end{aligned}
$$

To obtain a convenient representation of the decoding operator $\mathcal{D}$, we define the average recovery function as

$$
a_{i}=c_{d}^{\sum_{j=0}^{i-1} d^{j}} \varepsilon^{d^{i}}=\varepsilon_{0}\left(\frac{\varepsilon}{\varepsilon_{0}}\right)^{d^{i}}
$$

$$
R a v=\frac{1}{\left|R_{i}\right|} \sum_{i} R_{i}
$$


where the $R_{i}$ are the recovery operators (see Section II) Let $\mathcal{T} \in \mathcal{M}_{2^{n}, 2^{n}}$ be the diagonal matrix given by

$$
\mathcal{T}_{\sigma \sigma}=\eta(\operatorname{Rav}, \sigma)
$$

Where $\eta$ is the linear homomorphism defined in Section II (6). In particular note that if $\sigma$ commutes with all recovery operators $R_{i}$, then $\mathcal{T}_{\sigma \sigma}=1$, and if $\sigma$ anti-commutes with all of the recovery operators then $\mathcal{T}_{\sigma \sigma}=-1$. Then, from [1] we obtain for the decoding matrix

$$
\mathcal{D}=\mathcal{E}^{t} \mathcal{T}
$$

Lemma 5.6: The nonzero elements of $\mathcal{D}_{X}$ must be contained in $X_{S}$, and similarly for $Z$, although usually not for $Y$.

In particular this implies that if $\sigma=X$ or $\sigma=Z$, then $\mathcal{G}_{\sigma \sigma^{\prime}}$ depends only on $N_{\sigma I}, N_{\sigma X}, N_{\sigma Y}$, and $N_{\sigma Z}$. Then to find convergence of the $X$ and $Z$ rows, we can look at these rows separately.

Proof: Since $\mathcal{D}_{I}=I$, the non identity stabilizer elements must commute with half of the recovery operators. Only the nonzero elements of $\mathcal{D}_{\sigma}$ don't commute with exactly half of the recovery operators. This implies that each nonidentity element of $S(X)$ commutes with half of the elements of $R_{Z}=$ $R\left(\epsilon_{X}, Z\right)$, and similarly for $S(Z)$ and $R_{X}=R\left(\epsilon_{Z}, X\right)$. If half of either $R_{X}$ or $R_{Z}$ commute with some element of $S$, then half of all of the recovery operators commute with it. Now, pick some nonzero element $c=X^{\otimes n} s_{X} s_{Z}$ of $\mathcal{E}_{X}$, where $s_{i} \in S(i)$. If $c \notin A_{X}$ then $s_{Z} \neq I$. Then, if an element $r \in A_{X}$, it follows that $\eta(r, c)=\eta\left(r, s_{Z}\right)$, and so, half of $R_{X}$ commutes with $c$. Then, $c$ must commute with half of the recovery elements, and so must be zero in $\mathcal{D}_{X}$. Then the nonzero elements of $\mathcal{D}_{X}$ are in $A_{X}$.

Theorem 5.7: There exists functions $f_{1}(a, b, c, d)$ and $f_{2}(a, b, c, d)$ such that the following is true for $\mathcal{G}=\Omega^{c}\left(\mathcal{N}^{\otimes n}\right)$ :

$$
\begin{aligned}
\mathcal{G}_{X I} & =f_{1}\left(\mathcal{N}_{X I}, \mathcal{N}_{X X}, i \mathcal{N}_{X Y}, \mathcal{N}_{X Z}\right) \\
\mathcal{G}_{X X} & =f_{2}\left(\mathcal{N}_{X X}, i \mathcal{N}_{X Y}, \mathcal{N}_{X Z}, \mathcal{N}_{X I}\right) \\
\mathcal{G}_{X Y} & =i^{n} f_{2}\left(i \mathcal{N}_{X Y}, \mathcal{N}_{X Z}, \mathcal{N}_{X X}, \mathcal{N}_{X I}\right) \\
\mathcal{G}_{X Z} & =f_{2}\left(\mathcal{N}_{X Z}, \mathcal{N}_{X X}, i \mathcal{N}_{X Y}, \mathcal{N}_{X I}\right) \\
\mathcal{G}_{Z I} & =f_{1}\left(\mathcal{N}_{Z I}, \mathcal{N}_{Z X}, i \mathcal{N}_{Z Y}, \mathcal{N}_{Z Z}\right) \\
\mathcal{G}_{Z X} & =f_{2}\left(\mathcal{N}_{Z X}, i \mathcal{N}_{Z Y}, \mathcal{N}_{Z Z}, \mathcal{N}_{Z I}\right) \\
\mathcal{G}_{Z Y} & =i^{n} f_{2}\left(i \mathcal{N}_{Z Y}, \mathcal{N}_{Z Z}, \mathcal{N}_{Z X}, \mathcal{N}_{Z I}\right) \\
\mathcal{G}_{Z Z} & =f_{2}\left(\mathcal{N}_{Z Z}, \mathcal{N}_{Z X}, i \mathcal{N}_{Z Y}, \mathcal{N}_{Z I}\right) .
\end{aligned}
$$

Furthermore, these functions $f_{1}(a, b, c, d)$ and $f_{2}(a, b, c, d)$ are symmetric under permutations of $b, c$, and $d$.

Proof: The permutation $X \rightarrow i Y \rightarrow Z \rightarrow X$, sends $\mathcal{E}_{I}=S$ to itself, and sends

$$
\mathcal{E}_{X} \rightarrow i^{n} \mathcal{E}_{Y} \rightarrow \mathcal{E}_{Z} \rightarrow \mathcal{E}_{X}
$$

Then, from Lemma 5.6, and the fact that $X \leftrightarrow Z$ sends $\mathcal{D}_{X} \leftrightarrow$ $\mathcal{D}_{Z}, f_{1}$ and $f_{2}$ must exist as stated.

As for the symmetries, $\mathcal{G}_{X I}$ depends on $\mathcal{D}_{X}$ and $\mathcal{E}_{I}$. By permuting $X, i Y$, and $Z$, we preserve the stabilizer elements which are the nonzero elements of $\mathcal{E}_{I}$, and so $\mathcal{G}_{X I}$ is fixed under permutations of $N_{X X}, i N_{X Y}, N_{X Z} \cdot \mathcal{G}_{X X}$ depends on $\mathcal{D}_{X}$, and
$\mathcal{E}_{X}$. By permuting $I, Z$, and $i Y$, we preserve the nonzero elements of $\mathcal{E}_{X}$, which are $\bar{X}$ times the elements of $\mathcal{E}_{I}$ (see (17)), and so $\mathcal{G}_{X X}$ is fixed under permutations of $N_{X I}, i N_{X Y}$, and $N_{X Z}$. The other cases follow similarly.

Lemma 5.8: Let $\sigma \neq \sigma^{\prime}$ be single qubit Pauli matrices and let $\sigma^{\prime \prime}$ be a nonzero element of $\mathcal{E}_{\sigma}$. Then $\sigma^{\prime}$ appears tensored an even number of times in $\sigma^{\prime \prime}$.

Proof: In the case where $\sigma=I, \mathcal{E}_{\sigma}$ corresponds to the stabilizer group. Since $S$ is generated by even weight elements in $X_{S}$ and even weight elements in $Z_{S}$, in order for it to be Abelian, it must have the above property. For general $\sigma$ we have $\mathcal{E}_{\sigma}=\bar{\sigma} S$, and, using $\bar{\sigma}$ is $\sigma$ on all qubits, the desired result follows.

Theorem 5.9: A CSS code takes a channel $\mathcal{N}$ to the identity channel if and only if both vectors $\left[\mathcal{N}_{X I}, \mathcal{N}_{X X}, \mathcal{N}_{X Z}, i \mathcal{N}_{X Y}\right]$ and $\left[\mathcal{N}_{Z I}, \mathcal{N}_{Z Z}, \mathcal{N}_{Z X}, i \mathcal{N}_{Z Y}\right]$ converge to $[0,1,0,0]$ under the map

$$
\begin{array}{r}
{[a, b, c, d] \rightarrow\left[f_{1}(a, b, c, d), f_{2}(b, c, d, a),\right.} \\
\left.f_{2}(c, d, a, b), i f_{2}(d, a, b, c)\right] .
\end{array}
$$

In fact, it is sufficient that they converge to $[*, 1, *, *]$.

Proof: Obviously, this is a necessary condition. From Lemma 5.8, we see that each of the variables $b, c$, and $d$ in $f_{1}(a, b, c, d)$, and $f_{2}(a, b, c, d)$ must appear an even number of times in each term. So we may ignore any -1 sign in front of $\mathcal{G}_{X Y}$ or $\mathcal{G}_{Z Y}$. From the symmetries we have, it then follows that the aforementioned map determines convergence on the $X$ and $Z$ rows. The rest of the theorem follows from Theorem 5.4.

Remark (Unital Channels): In the case of unital channels, the above reduces to the condition that both $\left[\mathcal{N}_{X X}, \mathcal{N}_{X Z}, i \mathcal{N}_{X Y}\right]$ and $\left[\mathcal{N}_{Z Z}, \mathcal{N}_{Z X}, i \mathcal{N}_{Z Y}\right]$ converge to $[1, *, *]$ under the map

$$
[a, b, c] \rightarrow\left[f_{2}(a, b, i c, 0), f_{2}(b, i c, a, 0), i f_{2}(i c, a, b, 0)\right] .
$$

Notice that this no longer depends on $f_{1}$.

Lemma 5.10: For CSS codes, we have $\max \left(c_{d}, c_{m}\right) \leq$ $2^{(3 / 2)(n-k)}$ for $c_{d}$ and $c_{m}$ as defined in Theorem 5.5.

Proof: We use the bound of Theorem 5.5 for the nondiagonal terms. In the case of a CSS code, we have for $A=X$ or $A=Z$ that $\mathcal{D}_{A} \subset A_{S}$, and so the nonzero entries are given by $S(A) A^{\otimes n}$. Therefore, the sum in (14) has only $2^{(n-k) / 2}$ entries, giving an overall coefficient of $2^{(3 / 2)(n-k)}$.

1) Doubly-Even CSS Codes: Doubly even CSS codes are CSS codes that have weight divisible by four for $S(X)$ and $S(Z)$. For these codes we can strengthen Theorem 5.9. Define functions $g_{1}$ and $g_{2}$ that are the same as the $f_{1}$ and $f_{2}$ defined in Theorem 5.7, without the factors of $i$.

Theorem 5.11: A doubly even CSS code takes a channel $\mathcal{N}$ to the identity channel if an only if both $\left[\mathcal{N}_{X I}, \mathcal{N}_{X X}, \mathcal{N}_{X Z}, \mathcal{N}_{X Y}\right]$ and $\left[\mathcal{N}_{Z I}, \mathcal{N}_{Z Z}, \mathcal{N}_{Z X}, \mathcal{N}_{Z Y}\right]$ converge to $[0,1,0,0]$ under the map

$$
\begin{aligned}
& {[a, b, c, d] \rightarrow\left[g_{1}(a, b, c, d), g_{2}(b, c, d, a),\right.} \\
&\left.g_{2}(c, d, a, b), g_{2}(d, a, b, c)\right] .
\end{aligned}
$$

Proof: The stabilizer group is formed by generators $\in X_{S}$, and generators $\in Z_{S}$, each with weight divisible by 
4. Then $X$ and $Z$ together appear a number of times divisible by 4 in each stabilizer element (and similarly for $\{X, Y\}$, $\{Y, Z\})$. Following similar reasoning to that of the proof of lemma 5.8, we find that $c$ and $t$ together appear a divisible by four number of times in each term of $f_{j}(j=1,2)$. Then, $f_{j}(a, b, c, i d)=f_{j}(a, b, i c, d)$, and by definition

$$
g_{j}(a, b, c, d)=f_{j}(a, b, c, i d) .
$$

These $g_{j}$ satisfy all the symmetries above and the convergence relations of Theorem 5.9. (without the factors of $i$ ).

2) Example: [[7,1,3]] CSS Code: We use the example of the $[[7,1,3]]$ code, a doubly even CSS code commonly used in fault tolerance calculations, to illustrate how to find the functions defined in Theorem 5.7. and use Theorem 5.9 to analyze the convergence of channels under this code.

b) Computation of the Coding Map: The stabilizer group of this code is generated by the elements $I I I X X X X$, $I X X I I X X, X I X I X I X$ and IIIZZZZ, IZZIIZZ, $Z I Z I Z I Z$. Using the notation from Section $\mathrm{V}-\mathrm{C}$, the nonzero elements of $\mathcal{E}_{I}$ are the stabilizer group elements

$$
\begin{aligned}
\mathcal{E}_{I}= & \sum_{s \in S} s \\
= & (I I I I I I I+I I I X X X X)(I I I I I I I+I X X I I X X) \\
& \times(I I I I I I I+X I X I X I X)(I I I I I I I+I I I Z Z Z Z) \\
& \times(I I I I I I I+I Z Z I I Z Z)(I I I I I I I+Z I Z I Z I Z)
\end{aligned}
$$

We have $\bar{X}=X X X X X X X$, and $\bar{Z}=Z Z Z Z Z Z Z$. One notices that there are 7 terms that are some permutation of $I I I X X X X$. Let $p_{7}(I I I X X X X)$ denote the sum over these permutations. $p_{7}(I I I Y Y Y Y)$ and $p_{7}(I I I Z Z Z Z)$ give us the corresponding permutations of IIIYYYY and IIIZZZZ. Similarly, there are 42 terms that are $-I Z Z X X Y Y$, up to some permutation, so we define a function $p_{42}(-I Z Z X X Y Y)$ to sum over these. Then we can write

$$
\begin{aligned}
\mathcal{E}_{I}=I I I I I I I & +p_{42}(-I Z Z X X Y Y) \\
& +p_{7}(I I I X X X X+I I I Y Y Y Y+I I I Z Z Z Z) .
\end{aligned}
$$

With $\mathcal{E}_{\sigma}=\mathcal{E}_{I} \bar{\sigma}$ we get

$$
\begin{aligned}
\mathcal{E}_{X}= & X X X X X X X+p_{42}(-X Y Y I I Z Z) \\
& +p_{7}(X X X I I I I+X X X Z Z Z Z+X X X Y Y Y Y) \\
\mathcal{E}_{Y}= & -Y Y Y Y Y Y Y-p_{42}(-Y X X Z Z I I) \\
& -p_{7}(Y Y Y Z Z Z Z+Y Y Y I I I I+Y Y Y X X X X) \\
\mathcal{E}_{Z}= & Z Z Z Z Z Z Z+p_{42}(-Z I I Y Y X X) \\
& +p_{7}(Z Z Z Y Y Y Y+Z Z Z X X X X+Z Z Z I I I I) .
\end{aligned}
$$

The recovery operators which depend on $X$ are

$$
\begin{array}{r}
R\left(\varepsilon_{Z}, X\right)=\{I I I I I I, X I I I I I I, I X I I I I I, I I X I I I I \\
I I I X I I I, I I I I X I I, I I I I I I X I, I I I I I I X\} .
\end{array}
$$

Combining these with the recovery operations in $R\left(\varepsilon_{X}, Z\right)$, we easily find all 64 recovery operators. There are one in the form $I I I I I I I$, all seven permutations of $I I I I I I X$, all seven permutations of $I I I I I I Y$, all seven permutations of $I I I I I I Z$, and all
42 permutations of $I I I I I X Z$. (19) now allows us to find the elements of $\mathcal{D}_{\sigma}$. We calculate $\mathcal{D}_{X}$ from $\mathcal{E}_{X} . X X X X X X X$ commutes with 8/64 recovery elements, $X X X I I I I$ commutes with 40/64 recovery elements, and $X X X Z Z Z Z, X X X Y Y Y Y$, and $X Y Y I I Z Z$ each commute with $32 / 64$ of the recovery elements. Then

$$
\begin{aligned}
\mathcal{D}_{X}= & \frac{1}{4} p_{7}(X X X I I I I)-\frac{3}{4} X X X X X X X \\
= & \frac{1}{4} X X X I I I I+\frac{1}{4} X I I X X I I+\frac{1}{4} I X I X I X I \\
& +\frac{1}{4} I I X I X X+\frac{1}{4} I I X X I I X+\frac{1}{4} I X I I X I X \\
& +\frac{1}{4} X I I I I X X-\frac{3}{4} X X X X X X X .
\end{aligned}
$$

A similar calculation shows that $\mathcal{D}_{Z}=-(3 / 4) Z Z Z Z Z Z Z+$ $(1 / 4) p_{7}(Z Z Z I I I I)$, but $\mathcal{D}_{Y}$ does not follow this pattern.

Now, we wish to compute $\mathcal{G}_{X I}$. First, we look at how the $(1 / 4) p_{7}(X X X I I I I)$ component of $\mathcal{D}_{X}$ contributes. From $N_{I \sigma}=\delta_{I \sigma}$, it follows that only elements in $\mathcal{D}_{I}$ that are identity on the last four qubits contribute. This is just $I I I I I I I$, so we get $p_{7}\left((1 / 4) N_{X I} N_{X I} N_{X I} N_{I I} N_{I I} N_{I I} N_{I I}\right)=(7 / 4) N_{X I}^{3}$. For the $-(3 / 4) X X X X X X X$ component of $\mathcal{D}_{X}$, everything in $\mathcal{E}_{I}$ contributes. This gives a contribution of

$$
\begin{gathered}
-\frac{3}{4}\left(N_{X I} N_{X I} N_{X I} N_{X I} N_{X I} N_{X I} N_{X I}\right. \\
+p_{7}\left(N_{X I} N_{X I} N_{X I} N_{X X} N_{X X} N_{X X} N_{X X}\right. \\
\quad+N_{X I} N_{X I} N_{X I} N_{X Y} N_{X Y} N_{X Y} N_{X Y} \\
\left.\quad+N_{X I} N_{X I} N_{X I} N_{X Z} N_{X Z} N_{X Z} N_{X Z}\right) \\
\left.+p_{42}\left(-N_{X I} N_{X Z} N_{X Z} N_{X X} N_{X X} N_{X Y} N_{X Y}\right)\right)
\end{gathered}
$$

Together, these give

$$
\begin{array}{r}
\mathcal{G}_{X I}=\frac{7}{4} N_{X I}^{3}-\frac{3}{4}\left(N_{X I}^{7}+7 N_{X I}^{3}\left(N_{X X}^{4}+N_{X Y}^{4}+N_{X Z}^{4}\right)\right. \\
\left.-42 N_{X I} N_{X X}^{2} N_{X Y}^{2} N_{X Z}^{2}\right)
\end{array}
$$

A similar calculation shows that

$$
\begin{aligned}
& \mathcal{G}_{X X}=\frac{7}{4} N_{X X}^{3}-\frac{3}{4}\left(N_{X X}^{7}+7 N_{X X}^{3}\left(N_{X I}^{4}+N_{X Z}^{4}+N_{X Y}^{4}\right)\right. \\
& \left.-42 N_{X X} N_{X I}^{2} N_{X Z}^{2} N_{X Y}^{2}\right) \\
& -\mathcal{G}_{X Y}=\frac{7}{4} N_{X Y}^{3}-\frac{3}{4}\left(N_{X Y}^{7}+7 N_{X Y}^{3}\left(N_{X I}^{4}+N_{X X}^{4}+N_{X Z}^{4}\right)\right. \\
& \left.-42 N_{X Y} N_{X I}^{2} N_{X X}^{2} N_{X Z}^{2}\right) \\
& \mathcal{G}_{X Z}=\frac{7}{4} N_{X Z}^{3}-\frac{3}{4}\left(N_{X Z}^{7}+7 N_{X Z}^{3}\left(N_{X I}^{4}+N_{X Y}^{4}+N_{X X}^{4}\right)\right. \\
& \left.-42 N_{X Z} N_{X I}^{2} N_{X Y}^{2} N_{X X}^{2}\right) \text {. }
\end{aligned}
$$

For the functions $g_{j}$, which are related to $f_{j}$ by (20), we obtain

$$
\begin{aligned}
g(a, b, c, d): & =g_{1}(a, b, c, d)=g_{2}(a, b, c, d) \\
& =\frac{7}{4} a^{3}-\frac{3}{4} a^{7}-\frac{21}{4} a^{3}\left(b^{4}+c^{4}+d^{4}\right)+\frac{63}{2} a b^{2} c^{2} d^{2} .
\end{aligned}
$$

Note that

$$
\begin{aligned}
\mathcal{G}_{X I} & =g\left(N_{X I}, N_{X X}, N_{X Y}, N_{X Z}\right) \\
\mathcal{G}_{X X} & =g\left(N_{X X}, N_{X Y}, N_{X Z}, N_{X I}\right) \\
\mathcal{G}_{X Y} & =-g\left(N_{X Y}, N_{X Z}, N_{X I}, N_{X X}\right) \\
\mathcal{G}_{X Z} & =g\left(N_{X Z}, N_{X I}, N_{X X}, N_{X Y}\right) .
\end{aligned}
$$


c) Analysis: We consider the convergence of a row of the channel matrix $[a, b, c, d]$ as in Theorem 5.2. We have from Theorem 5.2 that

$$
a^{2}+b^{2}+c^{2}+d^{2} \leq 1
$$

If the channel is diagonal (or in general in the case where all but one parameter $a, b, c$ or $d$ are zero) we have a critical point $x_{c}=0.870807$ such that $g\left( \pm x_{c}, 0,0,0\right)= \pm x_{c}$.

Let us now analyze the behavior of nondiagonal channels with small off-diagonal elements.

Theorem 5.12: If any of $a, b, c$, or $d$ is within $x_{c}$ of 0 , it must go to 0 .

Proof: This can be proved in general by a rather lengthy calculation. To convey the main idea we will here only give the proof in the case where one of the 4 variables equals 0 (for example, a unital channel). Then our function $g$ becomes $g(a, b, c)=$ $(7 / 4) a^{3}-(3 / 4) a^{3}\left(a^{4}+7 b^{4}+7 c^{4}\right)$. We want to show that that if $0<|a|<x_{c}$, we have that $|a|>|g(a, b, c)|$. Without loss of generality, we may assume that $a$ is positive. Below the critical value $x_{c}$, we have $a>(7 / 4) a^{3}-(3 / 4) a^{7}$, so we only need to see if $a \leq-g(a, b, c)$, which is maximized by $b=$ $\sqrt{1-a^{2}}, c=0$. A simple calculation shows that there is no solution. Then it follows that $|a|$ must monotonically go to 0 .

From Theorem 5.12 and (21) we easily see that the vector $[a, b, c, d]$ must converge to a vector with at most one nonzero coefficient. Now suppose that $a$ is slightly above $x_{c}$, and that $b$, $c$, and $d$ have absolute values of at most some small $\epsilon$. We wish to see how much $\epsilon$ changes the critical convergence value for $a$. Let $k=\left.(d g(a, 0,0,0) / d a)\right|_{x_{c}}=1.691859$. Then,

$$
\begin{aligned}
g(a, b, c, d) & \geq g(a, \varepsilon, \varepsilon, \varepsilon) \\
& \geq g(a, 0,0,0)-\frac{63}{4} a^{3} \varepsilon^{4} \\
& \approx k\left(a-x_{c}\right)+x_{c}-\frac{63}{4} a^{3} \varepsilon^{4} .
\end{aligned}
$$

Since $b, c$, and $d$ become $O\left(\epsilon^{d}\right)=O\left(\epsilon^{3}\right)$ up to 4 th order of $\epsilon$, the vector converges to $[1,0,0,0]$ for $k\left(a-x_{c}\right)+x_{c}-(63 / 4) a^{3} \epsilon^{4} \geq$ $x_{c}$, which implies that

$$
k\left(a-x_{c}\right) \geq \frac{63}{4} a^{3} \varepsilon^{4} \approx \frac{63}{4} \varepsilon^{4}\left(x_{c}^{3}+3\left(a-x_{c}\right)^{2}\right) \approx \frac{63}{4} \varepsilon^{4} x_{c}^{3} .
$$

Solving up to first order for our new critical value, we get

$$
a=\frac{63 x_{c}^{3} \varepsilon^{4}}{4 k}+x_{c}=6.14726 \varepsilon^{4}+x_{c} .
$$

This implies that the off-diagonal terms affect the threshold to fourth order (as implied by Theorem 5.5.); but here we improved the prefactor $c_{t}$. Note that Lemma 5.10 would have given a prefactor of 512 .

If we choose a larger number instead of 6.14726 , for example 7 , then our vector converges to $[1,0,0,0]$ from $\left[x_{c}+7 \epsilon^{4}, \epsilon, \epsilon, \epsilon\right]$ for $\epsilon$ as big as 0.3 .

\section{SVD CANONICAL FORM}

In this section, we follow the method of [3], applying unitary gates before and after our channel to create a new channel that has fewer parameters. This can be used to improve the region of convergence to the identity channel.
Let $\sigma_{j}$ be the nonidentity elements of the Pauli group $\mathcal{P}$. Then if $U=e^{i(\theta / 2) \sigma_{1}}$, then the unitary channel $\rho \rightarrow U \rho U^{\dagger}$ performs a rotation by $\theta$ in the $\sigma_{3} \sigma_{2}$ plane. More generally:

Lemma 6.1: Expressing the unitary gates as channels in the Pauli basis creates a bijection from $S U(2) /( \pm I)$ to $1 \oplus S O(3)$

The Singular Value decomposition (SVD) theorem [12] states that if $A$ is a real matrix, then there exists $D=O_{2}^{\dagger} A O_{1}$ such that the $O_{i}$ are orthogonal, and $D$ is a diagonal matrix with elements $\lambda_{i} \geq 0$, which are called the singular values of $A$. Then $D=\operatorname{sgn}(\operatorname{det} A) R_{2}^{\dagger} A R_{1}$, where $R_{i} \in S O(n)$.

Theorem 6.2: If $\mathcal{N}^{(1)}$ is a channel on one qubit, then there exists a channel

$$
\mathcal{T}=\mathcal{U}_{2}^{\dagger} \mathcal{N}^{(1)} \mathcal{U}_{1}=\left(\begin{array}{cccc}
1 & 0 & 0 & 0 \\
t_{1}^{\prime} & \pm \lambda_{1} & 0 & 0 \\
t_{2}^{\prime} & 0 & \pm \lambda_{2} & 0 \\
t_{3}^{\prime} & 0 & 0 & \pm \lambda_{3}
\end{array}\right)
$$

where the $\mathcal{U}_{i}$ are channel representations of a matrix of $S U(2)$ in the form $1 \oplus S O(3)$, and the \pm designates the sign of $\operatorname{det} \mathcal{N}^{(1)}$.

d) Proof: From (3), define the vector $\mathbf{t}=$ $\left(N_{X I}, N_{Y I}, N_{Z I}\right)$, and let $A$ be the $3 \times 3$ matrix with the other nine variable elements. From the SVD theorem, we have

$$
\mathcal{T}=\left(\begin{array}{cc}
1 & 0 \\
0 & R_{2}^{\dagger}
\end{array}\right)\left(\begin{array}{cc}
1 & 0 \\
\mathbf{t} & A
\end{array}\right)\left(\begin{array}{cc}
1 & 0 \\
0 & R_{1}
\end{array}\right)=\left(\begin{array}{cc}
1 & 0 \\
\mathbf{t}^{\prime} & \pm D
\end{array}\right)
$$

where $\mathbf{t}^{\prime}=R_{2}^{\dagger} \mathbf{t}=\left(t_{1}^{\prime}, t_{2}^{\prime}, t_{3}^{\prime}\right)$. The outer matrices are unitary channels by lemma 6.1 .

Note that $\|\mathbf{t}\|=\left\|\mathbf{t}^{\prime}\right\|$, so if the channel is unital, $\mathbf{t}^{\prime}=\mathbf{0}$.

\section{A. CSS Codes}

We now apply the above to CSS codes, and in particular examine the [[7,1,3]] CSS code.

Proposition 6.3: For a given CSS code with a channel $\mathcal{T}$ in the canonical form of (22), if at least 2 of $\left[t_{i}^{\prime}, \lambda_{i}\right]$ converge to $[0,1]$ under the map

$$
[a, b] \rightarrow\left[f_{1}(a, b, 0,0), f_{2}(b, a, 0,0)\right]
$$

where the $f_{i}$ are the functions from Theorem 5.7 , then by applying unitary gates before and after the channel $\mathcal{T}$, we can create a new channel $\mathcal{T}^{\prime}$ that converges to the identity.

Proof: Suppose that $\left[t_{i}^{\prime}, \lambda_{i}\right]$ and $\left[t_{j}^{\prime}, \lambda_{j}\right]$ converge to $[0,1]$ under the given map. We define a matrix $A \in S O(4)$ such that $\sigma_{I} \rightarrow \sigma_{I}, \sigma_{i} \rightarrow \sigma_{X}, \sigma_{j} \rightarrow \sigma_{Z}$, and the diagonal matrix $B=$ $[1, \pm 1,1, \pm 1]$. By Lemma 6.1 , these are unitary channels. Then

$$
\mathcal{T}^{\prime}=A \mathcal{T} A^{\dagger} B=\left(\begin{array}{cccc}
1 & 0 & 0 & 0 \\
t_{i}^{\prime} & \lambda_{i} & 0 & 0 \\
* & 0 & * & 0 \\
t_{j}^{\prime} & 0 & 0 & \lambda_{j}
\end{array}\right)
$$

and the rest follows from Theorem 5.9.

Now, in a method similar to that of Theorem 5.9, we only need to consider the convergence of the two-dimensional real space $[a, b]=\left[t_{i}^{\prime} \lambda_{i}\right]$ under the map from (23). In particular, we are interested in the region of $[a, b]$ for which it converges to $[0,1]$. For the channel $\mathcal{T}^{\prime}$ from (24), if both $\left[t_{i}^{\prime}, \lambda_{i}\right]$ and $\left[t_{j}^{\prime}, \lambda_{j}\right]$ converge to $[0,1]$, then $\mathcal{T}^{\prime}$ converges to the identity channel. From Corollary 5.2, $a^{2}+b^{2} \leq 1$. 
1) Example: The [[7,1,3]] CSS Code: For the [[7,1,3]] code, the map is $h([a, b])=[g(a, b), g(b, a)]$, where $g(a, b)=\left(a^{3} / 4\right)\left(7-3 a^{4}-21 b^{4}\right)$. Let $\left[a_{n}, b_{n}\right]=h^{\circ n}([a, b])$. This converges to $[0,1]$ if and only if $b_{n} \rightarrow 1$.

A numerical calculation shows that $[a, b]$ always converges to $[0,1]$ for $b>b_{c} \approx 0.927334$. For $[a, b]=[\sin \theta, \cos \theta]$, this threshold is exact, and so this converges to $[0,1]$ for $|\theta|<\theta_{c} \approx$ 0.383572 . For a unital channel, $a=0$, and so this converges to $[0,1]$ for $b>x_{c} \approx 0.870807$. In either of these cases, we just need at most one singular value $\lambda_{i}$ of the channel to be less than or equal to the given critical value.

We can find an approximate solution for the region of convergence to $[0,1]$ by solving $b_{n} \geq x_{c}$. For $n=1$, we have an approximation for the region of $a^{4} \leq f_{1}(b)=(1 / 3)-(1 / 7) b^{4}-$ $\left(4 x_{c} / 21 b^{3}\right)$. As $n$ increases, these approximate regions rapidly converge to the actual region of convergence to $[0,1]$.

The singular values of a unitary channel are always 1 . Note that if the unitary channel $e^{i(\theta / 2) \sigma_{1}}$ from Lemma 6.1 is in its original non canonical form (we do not apply the unitary gates from Theorem 6.2), it converges to the identity channel for $|\theta|<\theta_{c}$.

\section{CONCLUSION AND FURTHER QUESTIONS}

\section{A. Drawbacks of Our Approach}

The approach of integrating the sequence of concatenated encoding and noise as a rather simple map from channels to channels is very powerful. By abstracting away from the details of the encoding and the noise process, it drastically reduces the number of parameters, and makes the coding process amenable to a dynamical systems type analysis. However, this approach sometimes comes at a price. By ignoring the details of the coding and correction process, we might get error thresholds above the actual thresholds if we accounted for all these details. The following example illustrates this, introducing the notion of a recovery function.

Suppose we have a $[[n, k, d]]$ stabilizer code. We define a recovery or error correcting function $R(\varepsilon)$ [13] which maps the collection of syndromes measured by the codes to some $n$ qubit Pauli operator, $R: \mathcal{F}_{2^{n-k}} \rightarrow \mathcal{P}_{n}$. We also define a syndrome function $\varepsilon: \mathcal{P}_{n} \rightarrow \mathcal{F}_{2^{n-k}}$, which maps Pauli errors to some syndrome. With these definitions we must have that $\beta=\varepsilon(R(\beta))$, for any $\beta \in \mathcal{P}_{n}$. Note that we can chose $R(\beta)$ up to elements of the stabiliser $S$ without any difference for error correction. Hence our choices for $R(\beta)$ differ from each other by elements of the centralizer $C(S)$ are limited to the $4^{k}$ elements of the Centralizer modulo the Stabilizer. They can be written as an element of $C(S)$ times some representative element of $S$. To study the choice of recovery function on the channel, define the matrix $T^{\sigma} \in \mathcal{M}_{4^{n}, 4^{n}}$ to be the diagonal matrix

$$
T_{\sigma^{\prime} \sigma^{\prime}}^{\sigma}=\frac{1}{2^{n-k}} \eta\left(\sigma, \sigma^{\prime}\right)
$$

Then the matrix operator $\mathcal{T}$, defined in (18), is $\mathcal{T}=\sum_{i} T^{R_{i}}$. We have $\mathcal{G}=\sum_{i} \mathcal{G}^{R_{i}}=\Omega^{C}(\mathcal{N})$, where the quasichannel (they do not have to preserve trace)

$$
\mathcal{G}^{R_{i}}=\mathcal{E}^{t} \mathcal{T}^{R_{i}} \mathcal{N} \mathcal{E}
$$

is the contribution of a single $R_{i}$ on the channel map.
When we measure a syndrome $\varepsilon$ during error-correction, we gain some information about the channel. Let the encoded state be described by the density matrix $\rho=\rho_{I} I+\rho_{X} X+\rho_{Y} Y+$ $\rho_{Z} Z$. We can re-write our channel $\mathcal{G}=\Omega^{C}(\mathcal{N})$ as a sum over all syndromes

$$
\mathcal{G}^{\prime}=\sum_{\beta \in F_{2^{n-k}}} \mathcal{G}^{R(\beta)} \otimes|\beta\rangle .
$$

If we measure $|\beta\rangle$ and use the information, we collapse to a syndrome $\beta$ with probability $p_{\beta}=\operatorname{tr}\left(\mathcal{G}^{R(\beta)} \rho\right)=2 \sum_{\sigma} \mathcal{G}_{I \sigma}^{R(\beta)} \rho_{\sigma}$, and the resulting density matrix is $\left(1 / p_{\beta}\right) \mathcal{G}^{R(\beta)} \rho$. In particular, if $\mathcal{G}_{I X}^{R(\beta)}=\mathcal{G}_{I Y}^{R(\beta)}=\mathcal{G}_{I Z}^{R(\beta)}=0$, then $p_{\beta}=2 \mathcal{G}_{I I}^{R(\beta)} \rho_{I}=$ $\mathcal{G}_{I I}^{R(\beta)}$, which doesn't depend on $\rho$, and the resulting $\rho$-independent channel is then $\left(1 / p_{\beta}\right) \mathcal{G}^{R(\beta)}$. If we throw this information away we recover the coding map $\mathcal{G}$ from the previous sections. In other words the coding map approach corresponds to ignoring the information about the channel that we could have obtained from the syndrome measurements, to optimize the recovery functions.

By performing measurements on the subblocks of a concatenated code, we affect the channel on each qubit of the top level code. If we do not optimize our error correction, we are not being as efficient as we should be. For example, a distance 3 code cannot correct some 2-qubit errors, and so the code we obtain by concatenating it once with itself without changing the error correction function cannot fix some 4-qubit errors. However, the distance $d$ of a distance $d_{1}$ code concatented with a distance $d_{2}$ code is $d \geq d_{1} d_{2}$, and so we should be able to correct any 4 qubit error. The problem is to keep track of all of this syndrome information, and finding the optimal error correction function seems to be computationally hard.

\section{B. Open Questions}

We have initiated a dynamical systems approach to quantum error correction, extending the result of Rahn et al.. [1]. This only opens the road to further analysis and many questions remain open. We list a few of them here.

In our analysis we have always assumed that an error correction process is successful, if the associated coding map takes the noise channel to the identity channel. However, this might be too stringent a condition. Are there any other criteria for information retrieval, which are not equivalent to zero (corrected) error?

Another question relates to the basin of correctable noise for a code: If our noise channel lies outside the basin of attraction of a certain code, can we find another code that would "lift" this noise into the basin of attraction of the old code? More specifically, given a code $C$ (with $d \geq 3$ ) and a noise channel $p \in \Delta-\mathcal{B}_{C}$, is there another code $C^{\prime}$ such that $\Omega^{C^{\prime}}(p) \in \mathcal{B}_{C}$ ? If the answer is positive, then the concatenation scheme $C^{k} \circ C^{\prime}$ corrects $p$, as $k \rightarrow \infty$. It would be interesting to formalize these ideas.

Yet another question concerns the shape of the region of correctable noise. Is there a (nontrivial) bound for the size or shape of the domain of attraction? Can we characterize regions of noise that are not correctable by any code? There is a new and interesting bound on noise from which no circuit can recover in 
[14]. However the methods used there are not dynamical. Is it possible to make sharper statements?

\section{ACKNOWLEDGMENT}

The authors would like to thank B. Whaley for support and fruitful conversations.

\section{REFERENCES}

[1] B. Rahn, A. C. Doherty, and H. Mabuchi, "Exact perfomance of concatenated quantum codes," Phys. Rev. A, vol. 66, no. 032 304, 2002.

[2] M. Nielsen and I. Chuang, Quantum Computation and Quantum. Cambridge, U.K.: Cambridge Univ. Press, 2000.

[3] C. King and M. B. Ruskai, "Minimal entropy states emerging from noisy quantum channels," IEEE Trans. Inform. Theory, vol. 47, no. 1, pp. 192-209, Jan. 2001.

[4] B. Hasselblatt and A. Katok, A First Course in Dynamics. Cambridge, U.K.: Cambridge Univ. Press, 2003.

[5] J. Palis and W. de Melo, Geometric Theory of Dynamical Systems. New York: Springer-Verlag, 1982.

[6] Quantum error correction fails for Hamiltonian models, R. Alicki. (2004). [Online]. Available: http://xxx.lanl.gov/abs/quant-ph/0411008

[7] Lecture Notes, J. Preskill. (1998). [Online]. Available: http://www. theory.caltech.edu/people/preskill/ph229/

[8] D. Gottesman, "Theory of fault-tolerant quantum computation," Phys. Rev. A, vol. 57, p. 127, 1997.

[9] P. Shor, "Scheme for reducing decoherence in quantum memory," Phys. Rev. A, vol. 52, pp. 2493-2496, 1995.

[10] A. Steane, "Error correcting codes in quantum theory," Phys. Rev. Lett., vol. 77, p. 793, 1996

[11] A. Calderbank and P. Shor, "Good quantum error correcting codes exist," Phys. Rev. A, vol. 54, pp. 1098-1105, 1996.

[12] R. Bhatia, Matrix Analysis. New York: Springer-Verlag, 1997.

[13] Probabilistic quantum error correction, J. Fern and J. Terilla. (2002). [Online]. Available: http://xxx.lanl.gov/abs/quant-ph/0 209058

[14] An upper bound on the threshold quantum decoherence rate, A. Razborov. (2003). [Online]. Available: http://xxx.lanl.gov/abs/quant-ph/ 0310136

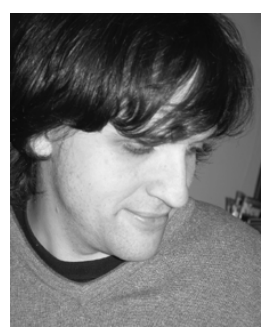

Jesse Fern received the B.S. degree in computer science, physics, and mathematics (unofficial) from the State University of New York, Stony Brook, in 2002. He is currently working toward the Ph.D. degree in mathematics at the University of California, Berkeley.

His research interests are quantum error correction and fault tolerance.

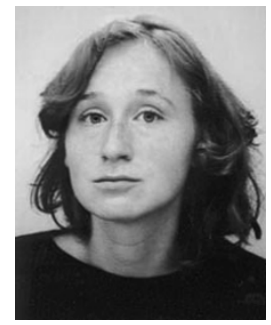

Julia Kempe received the B.S. degrees in mathematics and physics from the University of Vienna, Vienna, Austria, in 1995, and the Ph.D. degree in mathematics from the University of California, Berkeley, and in computer science from the Ecole Nationale Superieure de Telecommunications, Paris, France, both in 2001

She joined CNRS in 2001 and works at the Computer Science Department of the University of Paris, Orsay, France. She has held visiting appointments in computer science and mathematics at the University of California, Berkeley and MSRI, in 2002, 2003, and 2005. Her main research interests are quantum computation and information.

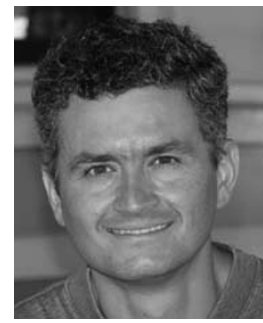

Slobodan N. Simić received the B.S. degree from the University of Belgrade, (former) Yugoslavia, in 1988, and the Ph.D. degree from the University of California, Berkeley, in 1995, both in mathematics.

After visiting appointments in mathematics and electrical engineering at the University of Illinois, Chicago, University of Southern California, Los Angeles, and the University of California, Berkeley. $\mathrm{He}$ joined San Jose State University, San Jose, CA, as an Assistant Professor of mathematics in 2004. and geometric control theory. His main research interests are dynamical systems

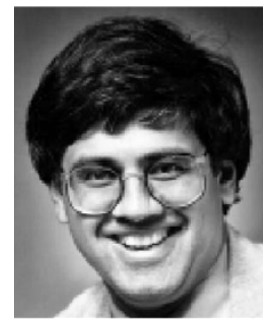

Shankar Sastry (F'94) received the M.A. degree (honoris causa) from Harvard University, Cambridge, MA, in 1994, and the Ph.D. degree from the University of California, Berkeley, in 1981.

$\mathrm{He}$ was on the faculty of Massachusetts Institute of Technology (MIT), Cambridge, as an Assistant Professor from 1980 to 1982 and Harvard University as a chaired Gordon Mc Kay professor in 1994. He served as Chairman of the Electrical Engineering and Computer Science (EECS) Department, University of California, Berkeley from 2001 to 2004. In 2000, he served as director of the Information Technology Office at DARPA. He is the NEC Distinguished Professor of EECS and a Crofessor of bioengineering and currently serves as the Director of Center for Information Technology in the Interests of Society (CITRIS). He has coauthored more than 300 technical papers and nine books.

Dr. Sastry received the President of India Gold Medal in 1977, the IBM Faculty Development award for 1983-1985, the US National Science Foundation Presidential Young Investigator Award in 1985, the Eckman Award of the American Automatic Control Council in 1990, the distinguished Alumnus Award of the Indian Institute of Technology in 1999, the David Marr prize for the best paper at the International Conference in Computer Vision in 1999, and the Ragazzini Award for Excellence in Education by the American Control Council in 2005. He is a member of the National Academy of Engineering and the American Academy of Arts and Sciences. He is on the US Air Force Science Board and is chairman of the Board of the International Computer Science Institute. He is also a member of the boards of the Federation of American Scientists and ESCHER (Embedded Systems Consortium for Hybrid and Embedded Research). 